that aims at improving care are that there is documentation of the work performed, a forum for discussing that documentation, and a system for taking action as a result of the discussion. The system for taking action implies that there is a formal committee structure within the health facility to receive information on audit and to make recommendations. There is published evidence that feedback of information to a clinical group and reaudit of activities shows altered management and improved care..$^{90}$ This is particularly so for the "procedural" specialties such as surgery, obstetrics and gynaecology, and anaesthesia. It is less easily demonstrated in internal medicine but therein lies the challenge for that "cognitive" specialty.

Finally, a successful audit programme requires a commitment on the part of both clinicians and administrators in healthcare facilities, and that commitment must be continuous because audit is never ending.

1 Pickering E. Accreditation survey findings. Australian Health Review 1980 3:15.

2 Renwick M, Harvey R. Quality assurance in hospitals: a digest. Australia: Australian Institute of Health, 1989.

3 Lembcke $P$. Medical auditing by scientific methods. FAMA 1956;162:646.

4 Smyth J. Surgical audit parts I and II. Med $\mathcal{F}$ Aust 1959;1:313-9.

5 Collopy BT. A surgical outcome audit. Med f Aust 1979;2:689-91.

6 Collopy BT, May J, Morgan B, Torr S. The results of an audit of primary appendicectomy in two Australian states. Aust Clin Rev 1983;10:6-10.

7 Cass M, Brook CW. Quality assurance: a state perspective. Aust Clin Rev 1990;10:129-31.

8 Collopy BT. Developing clinical indicators. The ACHS Care Evaluatio Program. Aust Clin Rev 1990;10:83-5.

9 Collopy BT. Quality assurance: much ado-much to do. Aust Clin Rev 1990;10:141-4.

10 Paul J. Quality assurance: its effect in practice. Aust Clin Rev 1990;10:126-8.

\section{Interim guidelines on confidentiality and medical audit}

Conference of Medical Royal Colleges and their Faculties in the United Kingdom

Medical audit is primarily an educational activity and will be professionally led (HC(91)2, para 3$)$. It is designed to improve the standards of patient care. As part of the implementation of the National Health Service and Community Care Act 1990 all doctors working in the NHS are required to undertake medical audit and, while recognising the need for confidentiality, it is required that managers and health authorities are provided with regular reports $(\mathrm{HC}(91) 2$, para 4). Potential conflicts of interest, therefore, arise in relation to the data required for medical audit and the needs of patients and clinicians for confidentiality and the need of management for information.

The Data Protection Act 1984 already allows patient access to data held on computers and word processors. The Access to Health Records Act 1990 will allow such access to manual records by the end of 1991. It is possible that medical audit data will be considered part of the medical record. Such records are likely to be discoverable at law in relation to litigation conducted on behalf of patients' interests and also may be used by employing authorities for disciplinary purposes. The colleges have been advised that the only exception to discovery in relation to litigation would be that the disclosure of records was not in the public interest. Such protection seems to have been implied for large regional or national audits (for example, the confidential enquiries into maternal and perioperative deaths), but has never been tested in the courts. The audit records of individual clinicians and units are almost certainly discoverable. No record of an audit meeting should contain any information that could allow identification of patients or clinicians or other hospital staff.

There are particular problems relating to issues of medical audit and confidentiality for patients, clinicians, and management which need separate consideration.

Patients-The confidentiality of all personal health information has been recently emphasised (NHS/90) (GEN/22). Usually patient consent is implicit, or explicit consent is obtained before passing on such information to other health professionals. This will not generally apply to medical audit. The necessity to anonymise patient data related to audit meetings is, therefore, emphasised. Only aggregated data or general conclusions should be passed on to management or to health authorities, to ensure that individual patients cannot be identified (HC(91)2, para 6e). However, the Audit Commission has rights of access to such information as it thinks necessary for audit without consent of patients or clinicians.

Clinicians-To achieve the goals of improved patient care and professional education, open and frank discussion during peer review or medical audit meetings is essential. The likelihood of discoverability of the records of such meetings poses a difficult problem for clinicians. All records of audit meetings, written or computerised, must be anonymised. There is no need to retain working protocols or proformas used for recording data from patient records as they duplicate information already available in the primary medical record. Serious problems relating to patient care identified by medical audit should be dealt with within the established professional procedures.

Management - The primary educational aims of medical audit in improving the overall standard of patient care rather than attempting to identify "bad apples" should be emphasised. Management needs to ensure that adequate medical audit procedures are in place, involving al doctors, and that the activity is both efficient and effective. It is the responsibility of local managers to ensure that adequate resources are available to support the agreed audit programme, together with the associated educational and training programmes. Support staff and appropriate information systems will be necessary in all units (HC(91)2, para 14). The requirements of confidentiality for both patients and clinicians mean that regular reports of audit activities to management must be anonymised. The reports should cover the general areas of activity audited, the overall conclusions and recommendations made, and plans for action or procedural changes, the necessity for which has been revealed by the audit (HC(91)2, para 8). There should also be a record of when a review of the results of the changes should be made and the proposed methods of review. These reports will normally be submitted to management through the medical audit committee.

These interim guidelines have been endorsed by the chief medical officer of the Department of Health. The conference is grateful to Drs Peter Beck and Anthony Hopkins for their help in preparing this guidance.

\section{Model minutes for medical audit meetings}

Conference of Medical Royal Colleges and their Faculties in the United Kingdom

Anonymity: No record of any patient's name or other identifying information should be made. There is no need to retain working documents used in the audit process, as they duplicate material already in the medical records.

1 Date and place of meeting.

2 Names, health service grade, and specialty of those attending.

3 (a) Cases considered:

(i) by diagnosis

(ii) by ICD (ninth revision) code.

(b) Specific problems considered:

(i) clinical

(ii) organisational.

4 Lessons arising from audit.

5 Plan of action.

6 Nominated individual to take action on this topic.

7 Review of progress on plans for action on earlier topics.

8 Date of next meeting. 